\title{
EL DESARROLLO DE COMPETENCIAS LABORALES Y LA FORMACIÓN DE LOS PROFESIONALES EN TURISMO EN LA UNIVERSIDAD NACIONAL MAYOR DE SAN MARCOS, AÑO 2013
}

\author{
Mauro Amaru Granados Maguiño* \\ granadosmaurom@gmail.com
}

\begin{abstract}
RESUMEN
El objetivo de nuestra investigación es determinar la relación entre el desarrollo de las competencias laborales y la formación de los profesionales en Turismo de la Universidad Nacional Mayor de San Marcos, en el año 2013.

La investigación demuestra que del total de estudiantes analizados, el 54,17\% tiene una percepción buena sobre las competencias básicas, el $67,71 \%$ consideran que son buenas las competencias genéricas y el 58,33\% opinan que son buenas las competencias específicas.

Los docentes debemos tener en cuenta que el alumno debe adquirir conocimientos actualizados de las distintas áreas del turismo (universalidad). Además, debemos desarrollar en ello la capacidad de entender de forma sistemática la información relevante, su contexto y cómo aplicarla a situaciones complejas (dominio de la información), así como dominar las técnicas suficientes que le permitan obtener y analizar información, evaluar su relevancia y validez, sintetizarla y adaptarla al contexto (en tecnificación).
\end{abstract}

Palabras clave: Competencias Laborales, Formacion Profesional, Habilidades.

\begin{abstract}
The objective of our research is to determine the relationship between the development of work competencies, as well as the apprenticeship of tourism professionals of the National University of San Marcos during 2013.

The research demonstrates that of the analyzed students total, $54.17 \%$ has a good perception about basic competencies, $67.71 \%$ considers that generic competencies are good and $58.33 \%$ thinks that specific competencies are good.

We as professors should have into account that student must acquire updated knowledges of the variety of tourism areas (universality). In addition, we have to develop capacity to understand systematically relevant information, its context and how to apply this information to complex situations (information domain). Moreover, to domain enough technics to obtain and analyze information, to synthetize and adapt it according to the context in technification.
\end{abstract}

Keywords: Work Competencies, Apprenticeship, Habilities.

* Licenciado en Turismo y Hotelería - USMP, Mag. en Marketing Turístico y Hotelero - USMP, Doctor en Educación - UNMSM. Director de la Escuela Académica Profesional de Administración de Turismo-UNMSM 


\section{INTRODUCCIÓN}

- Normativa y legal: en lo normado en la Ley 23733, Ley Universitaria, en tanto es requisito la sustentación de un trabajo de investigación para la obtención del grado Académico de Doctor en Educación.

- Teórica: en la medida en que para fundamentar las variables del estudio (competencias laborales y la formación de los profesionales en Turismo), debemos sustentarnos en las fuentes teórico científicas mas actualizadas.

- En lo práctico: el presente estudio servirá para mejorar el proceso de formación profesional, en las competencias laborales de los futuros profesionales en turismo.

- Científica: el estudio permite la aplicación del método científico en todas sus etapas a fin de llegar a las conclusiones y recomendaciones o sugerencias que permitan mejorar el servicio que ofrece la Escuela Académico Profesional de Turismo de la Universidad Nacional Mayor de San Marcos (UNMSM).

La hipótesis es directa y significativa la relación entre el desarrollo de las competencias laborales y la formación de los profesionales en Turismo, en la Universidad Nacional Mayor de San Marcos, en el año 2013.

La investigación demuestra que del total de estudiantes analizados, el 40,63\% opina que son buenas las competencias cognoscitivas y el $58,3 \%$ opina que son buenas las habilidades y destrezas. La investigación demuestra que del total de estudiantes analizados el 59,38\% opina que son buenas las competencias laborales, el $50 \%$ opina que es regular y el $48,96 \%$ opina que es buena la formación de los profesionales en Turismo.

La investigación demuestra que el desarrollo de las competencias básicas y la formación en competencias cognoscitivas del perfil se relacionan significativamente, concluyendo que existe una correlación moderada positiva y significativa $(0,39)$ entre el desarrollo de las competencias básicas y la formación en competencias cognoscitivas del perfil de los profesionales en Turismo.

La investigación también demuestra también que el desarrollo de las competencias básicas y la formación en habilidades y destrezas del perfil se relacionan se relacionan significativamente, concluyendo que existe una correlación moderada positiva y significativa $(0,41)$ entre el desarrollo de las competencias básicas y la formación en habilidades y destrezas del perfil de los profesionales en Turismo.

La investigación demuestra que el desarrollo de las competencias genéricas y la formación en competencias cognoscitivas del perfil se relacionan se relacionan significativamente, concluyendo que existe una correlación moderada positiva y significativa $(0,47)$ entre el desarrollo de las competencias genéricas y la formación en competencias cognoscitivas del perfil de los profesionales en Turismo.

La investigación demuestra que el desarrollo de las competencias genéricas y la formación en habilidades y destrezas del perfil se relacionan significativamente, concluyendo que existe una correlación moderada positiva y significativa $(0,61)$, entre el desarrollo de las competencias genéricas y la formación en habilidades y destrezas del perfil de los profesionales en Turismo.

La investigación demuestra que el desarrollo de las competencias específicas y la formación en competencias cognoscitivas del perfil se relacionan significativamente, concluyendo que existe una correlación moderada positiva y significativa $(0,49)$, entre el desarrollo de las competencias específicas y la formación en competencias cognoscitivas del perfil de los profesionales en Turismo.

Finalmente la investigación demuestra que el desarrollo de las competencias específicas y la formación en habilidades y destrezas del perfil se relacionan se relacionan significativamente, concluyendo que existe una correlación moderada positiva y significativa $(0,59)$ entre el desarrollo de las competencias específicas y la formación en habilidades y destrezas del perfil de los profesionales en Turismo.

\section{METODOS}

Teniendo en cuenta lo expuesto por Mejía (2005), la investigación ha sido tipificada en la forma siguiente:

- Según el tipo de conocimiento previo en la investigación, la investigación es científica.

- Según la naturaleza del objeto de estudio, es factual o empírica. 
- Según el tipo de pregunta planteada en el problema, es teórica descriptiva relacional no causal.

- Según el método de contrastación de hipótesis es ex post facto.

- Según el método de estudio de las variables es cuantitativa.

- Según el número de variables es bivariada.

- Según el ambiente en que se realiza es bibliográfica y de campo.

- Según el tipo de datos que producen es secundaria.

- Según el enfoque utilitario predominante es teorética o especulativa.

- Según la profundidad con que se trata el tema es previa, piloto.

- Según el tiempo de aplicación de la variable, es transversal o sincrónico.

\section{DISCUSIÓN DE LOS RESULTADOS.}

Los cambios que las empresas atraviesan no se dejan caracterizar fácilmente, ya que el mundo es más competitivo, con mayor cantidad de participantes que buscan aumentar intervención en mercados compartidos por la creciente globalización. Es por eso que estas necesitan incorporar acciones que les permitan ser líderes en el mercado, y a sus trabajadores, obtener los conocimientos, habilidades y conductas para que alcancen el desempeño laboral superior, siendo competentes en sus ocupaciones, según los intereses de la organización y los clientes.

En el contexto actual de las competencias en el marco del capital humano, actualmente existe el término de competencia y competencia laboral de forma diferenciada, aunque con el mismo propósito: de lograr organizaciones y capital humano competentes que es el encuentro entre organizaciones y capital humano para el logro de la excelencia, donde las organizaciones y el capital humano busquen ser competentes.

Competencia laboral es el sistema de conocimientos, habilidades y conductas que incluye a todos los componentes de la configuración psicológica compleja, necesarios para la evaluación de la idoneidad del capital humano en el desempeño profesional del cargo que ocupa y de su vida personal. Como se puede apreciar, no se considera solo al capital humano competente (ya que de esta forma estaría definiendo el término de competente con resultado eficaz, el desempeño laboral superior y no el de competencia), sino también a aquellos que les faltan algunos de estos componentes, donde están presentes los meta cognitivos (autocontrol de conocimientos) y cognitivos (conocimientos), actitudes, motivacionales y otras cualidades de la personalidad, como deseos, estado de ánimo (conductas) ínter-vinculados como sistema y que algunos autores cubanos llaman configuración psicológica compleja. Ser competente dentro del capital humano exige un dominio técnico de conocimientos y acciones (habilidades) relacionado con las exigencias del modelo o perfil profesional que presupone la excelencia en la solución de los problemas, funciones principales o tareas concretas con un comportamiento o normas de conductas que permitan el logro de los objetivos propuestos por la organización. En este nuevo concepto, se considera a la capacidad dentro de la competencia laboral como capacidad potencial y no real, que puede ser o no competente pero que permite comprobar las funciones principales (conocimientos) del individuo en el desempeño profesional para alcanzar los resultados deseados, es decir, estar aptos para resolver o solucionar los problemas en el ejercicio de su ocupación y ejercer otras nuevas, según su modo de actuación en la vida laboral y personal.

La mejora en la capacitación y formación de los directivos de las instalaciones turísticas es una actividad de primordial importancia para conseguir un desempeño óptimo que coadyuve a un posicionamiento importante en el área del turismo.

La selección de profesionales se basa potencialmente en su integridad e incondicionalidad a los principios éticos, políticos de nuestra sociedad así como a conocimientos técnicos, pero donde todavía no se ha instaurado a profundidad todo lo relacionado con la concepción de competencias laborales demostradas en contexto y su posible pericia profesional.

Teniendo en cuenta que la formación es una de las vías para imbuir a la persona de las habilidades y conocimientos necesarios, así como de valores, el rendimiento institucional depende en gran medida de una óptima selección de necesidades formativas en los directivos. 
Los procesos formativos en nuestro país están cada vez mejor dotados de variedad de ofertas así como de medios, vías y métodos que redunden en resultados de excelencia en el educando, así como en las herramientas y tecnologías que viabilicen y enriquezcan el proceso.

La detección de necesidades formativas y el impacto de la formación son las dos dimensiones claves de cara a una buena estrategia en formación. Se trata de responder a las preguntas: ¿cuáles son los déficits de conocimientos y habilidades de los directivos de las empresas turísticas para conseguir la máxima eficacia y eficiencia institucional en el marco de un proceso de mejora continua?

La dimensión de la detección de necesidades es, seguramente, el punto más escabroso de todo el ciclo formativo. En la práctica se confunden percepciones subjetivas de los propios profesionales y de sus superiores, con debilidades organizativas más objetivas pero difíciles de definir.

Tradicionalmente, la detección de necesidades es más producto de la intuición de los especialistas en materia de formación que de una selección sistemática y de carácter científico. Este artículo incursiona en este reto y su objetivo es aportar criterios conceptuales e instrumentos metodológicos orientados a conseguir un mayor rigor en la detección de las necesidades formativas.

Para diseñar planes y estrategias de formación aplicables se requiere conocer muy bien los objetivos, potencialidades, particularidades y planeación estratégica quinquenal de la organización a la que se destina el proyecto, el contexto histórico-social en el que se desarrolla la organización, intereses individuales, así como el desarrollo alcanzado por los trabajadores en sus competencias laborales.

Las competencias laborales juegan un valor importante en el sector turístico, ya que mediante éstas el capital humano demuestra el dominio de los conocimientos, habilidades y conductas en la profesión que se desempeña, contribuyendo al mejoramiento de la eficiencia y eficacia del trabajo, realizando las cosas bien desde el inicio para la satisfacción de las necesidades y expectativas de los clientes internos y externos que los visitan.

Por eso, la gestión por competencias en el turismo está en la capacidad organizativa que tienen las instalaciones para la creación y el me- joramiento continuo de la calidad de su producto turístico, con el capital humano idóneo.

Para el análisis se aplicó el método cuantitativo. Para encontrar las relaciones se aplicó "en primera instancia" una prueba de normalidad por el método de Kolgomorv-Smirnov que nos permitió encontrar la normalidad de las variables en estudio así como sus dimensiones.

Luego se procedió encontrar la relación de las variables en estudio por el método de Chi Cuadrado y el grado de la asociación por el coeficiente de correlación de Pearson.

Para comprobar la primera hipótesis de investigación se encontró que el desarrollo de las competencias básicas y la formación en competencias cognoscitivas del perfil se relacionan de forma estadísticamente significativa $(\mathrm{P}<0,05)$

Existe una correlación moderada positiva y significativa $(0,39)$, entre el desarrollo de las competencias básicas y la formación en competencias cognoscitivas del perfil de los profesionales en turismo, resultado que coincide con la investigación.

Se tiene que el $54,17 \%$ y del total de encuestados considera que las competencias básicas son buenas y el $40,63 \%$ opina que son buenas las competencias cognoscitivas, resultado que contrastado con la investigación de Tito, Pereda, y Vilcabana, $(2008: 4,64,66,68 y 144)$ en cuyo resultado encontraron que los estudiantes manifestaron que los conocimientos impartidos en la universidad sirvieron poco o medianamente para su desempeño laboral óptimo. Sin embargo, coincide con la investigación de Torres y Merino, (2010), donde la percepción estudiantil con respecto a la ejecución curricular es poco satisfactoria también se equipará con la investigación de Lillo, Ramón y Sevilla (2006: 4,64,5) quien propone un modelo holístico para el análisis del capital humano aplicado al sector turístico que puede servir de base para el diseño de la formación turística en cualquier dimensión de esta actividad, sea un país, destino, empresa o subsector y puede ayudar a establecer los pasos a seguir en iniciativas eficaces de políticas de formación en las distintas organizaciones turísticas. Los estudiantes tienen buena información para desempeñarse en cualquier actividad productiva, tales como la aplicación de sistemas de procesamiento de datos, interpretación de la realidad económica, aplicar sistemas numéricos para análisis de datos, saber expresarse y saber escuchar. 
Los estudiantes poseen una gran ventaja dado que mantienen una buena percepción acerca de la competencia básica; es decir, disponen de una buena formación y gestión de sus conocimientos.

Para comprobar la segunda hipótesis de investigación se encontró que el desarrollo de las competencias básicas y la formación en habilidades y destrezas del perfil se relacionan de forma estadísticamente significativa $(\mathrm{P}<0,05)$.

Así mismo existe una correlación moderada positiva y significativa $(0,41)$ entre el desarrollo de las competencias básicas y la formación en habilidades y destrezas del perfil de los profesionales en turismo. Este resultado coincide con la investigación. Los resultados muestran que el 58,3\% opina que son buenas las habilidades y destrezas. Esta investigación coincide con la investigación de Robalino (2012: 11 y 157) y Torres y Merino, (2010), quienes aseveran que se hace imperiosa la necesidad de una mayor atención hacia los aspectos del mejoramiento de los medios y materiales para el desarrollo de los cursos. Se recomienda la implementación de un sistema de supervisión y evaluación curricular permanente. Los estudiantes están en capacidad y condiciones -de acuerdo al conjunto de conocimientos, habilidades, destrezas y valores que deben tener los egresados- para obtener su titulación y lograr el liderazgo profesional en el entorno laboral del sector turismo, el cual se viene consolidando como una de las principales actividades económicas del país y del mundo. En consecuencia, están capacitados para gerenciar empresas, organizaciones, e instituciones del sector turismo, con criterio innovador, técnico y enfocadas en los mercados nacionales e internacionales.

La tercera hipótesis de investigación demuestra que el desarrollo de las competencias genéricas y la formación en competencias cognoscitivas del perfil de los profesionales en turismo, $(\mathrm{P}<0,05)$. Por otro lado, se observa que existe una correlación moderada positiva y significativa $(0,47)$ entre el desarrollo de las competencias genéricas y la formación en competencias cognoscitivas del perfil de los profesionales en turismo. En este tipo de competencias hemos registrado aquellos conocimientos y habilidades que están asociados al desarrollo de diversas áreas y sub áreas ocupacionales; es decir, son las competencias que definen un perfil concreto para las distintas actividades. Por ejemplo, analizar y evaluar información, trabajar en equipo, planear acciones, coaching, deontología, liderazgo, desarrollo emocional, manejo de personal, adaptación, entre otras. Estos resultados coiciden con la investigación de Lillo, Ramón y Sevilla (2006: 4,6 y 15), García y Pérez (2008), Gómez, (2010: 46). La educación basada en competencias supone un compromiso de toda la comunidad -académicos, autoridades y alumnoscon y para el cambio. De hecho, por ejemplo, si no varían los mecanismos de evaluación que se han venido aplicando en la mayoría de los cursos de esta carrera, será un perfecto engaño valorar el desempeño de las competencias en los egresados.

Las principales razones para la inclusión de este tipo de competencias provienen, por un lado, de las instituciones y las empresas que contratan a los titulados universitarios, quienes demandan una formación basada en competencias para poder contar con recursos humanos que no solo posean competencias técnicas sino también competencias metodológicas, humanas y sociales; es decir, que cuenten con todas aquellas competencias relacionadas con la capacidad de actuación efectiva en las situaciones concretas de trabajo y en la vida, en general (Rychen y Salganik, 2001, 2003).

Por otro lado, la inclusión de estas competencias en la formación universitaria es lo que permite evitar una simplificación reductora de las posibilidades formativas de la universidad, no restringiéndose únicamente a las competencias profesionales específicas sino proporcionando una formación integral al individuo (Yáñiz, 2006)

El turismo es una actividad eminentemente humana que requiere de profesionales que investiguen, gestionen, administren, innoven y proporcionen servicios de calidad. La formación de estos profesionales y técnicos es función de universidades e institutos de educación superior que deben estar a la vanguardia de los conocimientos, tanto de la disciplina que imparten como de las nuevas tendencias educativas.

La administración del currículo a través de distintas propuestas pedagógicas ha sido un tema debatido por expertos en educación. La formación profesional a través del desarrollo de competencias está relacionada con la psicología industrial, la gestión del talento humano, y criticada porque se piensa que el estudiante pasa por un proceso de adiestramiento repetitivo que carece de reflexión y que no tiene otro fin que perfeccionar procesos hasta llegar a la automatización del individuo. 
La cuarta hipótesis de investigación demuestra que el desarrollo de las competencias genéricas y la formación en habilidades y destrezas del perfil se relacionan de forma estadísticamente significativa. Se observa que existe una correlación moderada positiva y significativa $(0,61)$ entre el desarrollo de las competencias genéricas y la formación en habilidades y destrezas del perfil de los profesionales en turismo. Lillo, Ramón y Sevilla (2006: 5, 6 y 15), consideran imprescindible pues, obtener una visión de conjunto de las interrelaciones, retos y necesidades de este factor en el sector turismo. Un polo de análisis importante es esa mirada al tejido productivo turístico que se establece a partir de la demanda de perfiles profesionales y el conocimiento de las necesidades reales de las empresas. También el entorno laboral turístico es objeto de análisis para establecer las normativas oportunas para un buen desarrollo de las políticas educativas turísticas. Así mismo, desde las instituciones educativas también se plantean decisiones que afectan a todos los ángulos analizados. En suma, este modelo holístico para el análisis del capital humano aplicado al sector turístico puede servir de base para el diseño de la formación turística en cualquier dimensión de esta actividad, sea un país, destino, empresa o subsector y puede ayudar a establecer los pasos a seguir en iniciativas eficaces de políticas de formación en las distintas organizaciones turísticas. También coincide con la investigación de García y Pérez (2008: 2, 3, 4, 6, 8, 10 y 16), Gómez, (2010: 1 y 10) y Vásquez, (2010: 8 y 36) quienes demuestran que los alumnos no han adquirido por completo las competencias buscadas en el plan de estudios, y no existe correlación en sus respuestas con la edad, experiencia laboral, puesto desempeñado, promedio obtenido hasta la fecha en la Licenciatura, y selección de la carrera.

El desarrollo en la formación universitaria formula un conjunto de competencias específicas, partiendo del cumplimiento de dos principios generales. El primero son las características especiales de los componentes de la titulación, alta formación para la dirección y gestión empresarial y capacitación para el acceso al doctorado, y como consiguiente formación para la investigación. El segundo principio son los objetivos de dichas competencias que han de tener la posibilidad de ser evaluables mediante procesos y métodos objetivos. En este sentido se han propuesto mecanismos y pruebas de carácter objetivo para hacer evaluables todas las competencias. Los es- tudiantes tiene una alta ccapacidad para alcanzar un conocimiento de lo que es la oferta, desde una perspectiva económica inversora nacional e internacional, así mismo el egresado tiene el control y medida de las variables turísticas para adquirir competencia en el tratamiento de la información estadística, así como la capacidad para interpretar y analizar proyectos de inversión, con un manejo técnico y alternativo de fuentes de financiación, consideración de las fuentes productivas y de sus ventas, y el cálculo de costos para llegar a la deducción de los conceptos y variables de beneficio o de rentabilidad. También pueden conocer los fundamentos y las herramientas para lograr la competitividad y eficacia, tanto en los productos como en los destinos

La quinta hipótesis de investigación demuestra que el desarrollo de las competencias específicas y la formación en competencias cognoscitivas del perfil se relacionan. Se observa que existe una correlación moderada positiva y significativa $(0,49)$ entre el desarrollo de las competencias específicas y la formación en competencias cognoscitivas del perfil de los profesionales en turismo. Estos resultados coinciden con la investigación de Torres y Merino (2010), y Tito (2012: 4, 64, 66, 68 y 144), y proponen una mejor distribución de planta en el proceso productivo, se elaboran el catálogo de competencias transversales para el sector y los perfiles para cada puesto tipo desde este enfoque. De la misma forma se propone una metodología de implantación del modelo en el sector. En ese orden de ideas, las recomendaciones pasan porque se instituya un proceso de formación y capacitación permanente de los cuadros directivos y técnicos, creando centros e institutos que tanto el sector privado y público deben asumir.

Según Martínez y Sauleda (2005: 9) el concepto competencia es "la capacidad de responder a demandas complejas y llevar a cabo tareas diversas de forma adecuada. Supone una combinación de habilidades prácticas, conocimientos, motivación, valores éticos, actitudes, emociones y otros componentes sociales y de comportamiento que se movilizan conjuntamente para lograr una acción eficaz". Estos autores profundizan en la definición y hacen referencia al contexto educativo y de evaluación, afirmando que el término competencia "se refiere a capacidades internas, destrezas, habilidades, dominio, prácticas o expertidad alcanzada por el aprendiz, por lo que precisa de una enseñanza centrada en el estudiante y de una evaluación que no tenga en cuenta solo los 
conocimientos de los contenidos curriculares, sino del saber hacer procedimental."

El turismo sigue siendo una de las actividades profesionales con más futuro. Sin embargo, pese a su importancia o tal vez debido a ello, el sector se encuentra en total evolución sufriendo los efectos de la globalización y de la innovación de los productos turísticos.

Todos estos cambios están teniendo su impacto en los recursos humanos del sector y en las competencias profesionales requeridas, siendo las competencias en Tecnologías de la Información y la Comunicación (TIC) y las competencias linguíísticas aquellas que parecen ser más demandadas por los empleadores. En esta línea van las aportaciones de Garrigós y Narangajavana (2006), los cuales afirman que el desarrollo de las tecnologías de la información y el impacto de Internet en el sector turístico han sido especialmente cruciales y virulentos. A la vez postulan que la influencia del entorno dinámico global y los rápidos avances en las tecnologías de la información durante la pasada década han incrementado el reconocimiento de que el conocimiento es el único recurso que puede facilitar una ventaja sostenible a las empresas.

La sexta hipótesis de investigación demuestra que el desarrollo de las competencias específicas y la formación en habilidades y destrezas del perfil se relacionan de forma estadísticamente significativa. Se observa que existe una correlación moderada positiva y significativa $(0,59)$, entre el desarrollo de las competencias específicas y la formación en habilidades y destrezas del perfil de los profesionales en turismo. Estos resultados coinciden con la investigación de Vásquez (2010: 8 y 36), Robalino (2012: 11 y 157), Tito, Pereda, y Vilcabana (2008: 4, 64, 66, 68, y 144), quienes revelan que existe una mediana correspondencia entre el ámbito de ocupación laboral definido por la universidad, a través de sus planes de estudios, y el nivel de empleabilidad y ocupabilidad de nuestros egresados en el mercado laboral. Tal afirmación se corrobora cuando el 53\% de los encuestados manifestaron que los conocimientos impartidos en la universidad sirvieron poco o medianamente para su desempeño laboral óptimo.

El capital humano reviste una importancia estratégica fundamental pues los factores intangibles prevalecen sobre los tangibles, y lograr una ventaja competitiva sostenible implica desarrollar estrategias de diferenciación que faciliten la vivencia de una experiencia auténtica e individualizada a los turistas. Desde este punto de vista, la gestión del capital humano y el desarrollo de competencias laborales constituyen aspectos claves para robustecer la competitividad turística, ya que es a través de ellas que se puede crear un ambiente laboral que permita desarrollar toda la capacidad y esfuerzo de los trabajadores en aras de maximizar la satisfacción de los visitantes.

Lograr este objetivo implica también que las empresas, los prestadores de servicios y demás operadores turísticos inviertan sostenidamente en la educación y capacitación de los recursos humanos que dan soporte a la actividad.

El movimiento hacia la adopción del enfoque de competencias laborales se ha relacionado con los cambios asociados a la globalización. Mertens (1997), asocia las competencias laborales con la generación de ventajas competitivas, la estrategia de productividad y la gestión del capital humano. Las empresas han entendido la necesidad de prevalecer en el mercado generando ventajas competitivas; para Mertens el problema se sintetiza en ¿cómo pueden diferenciarse las empresas en un mercado tendiente a globalizarse y que facilita la difusión rápida y masiva de mejores prácticas organizativas e innovaciones tecnológicas?

Las estrategias empresariales hacia el mejoramiento de la competitividad han generado elementos de diferenciación a partir de la estructura organizacional y de la incorporación de elementos que antes constituían su entorno. Así, se construyeron redes de colaboración entre las empresas y otros agentes clave como los proveedores, consultores, contratistas, clientes y trabajadores, donde lo importante no eran los activos físicos y financieros sino otros intangibles muy valiosos, como el conocimiento, la formación, la capacidad de innovación, el manejo del mercado, los sistemas de motivación, etc.

Habitualmente las organizaciones han empleado la definición de los puestos de trabajo para identificar los rasgos de las personas que podrían cubrirlos satisfactoriamente. En el enfoque de competencias se parte de las características y los comportamientos de las personas que realizan eficazmente las tareas correspondientes a un puesto de trabajo. Esto significa concentrarse en comportamientos observables que son consecuencia de un conjunto de motivaciones, rasgos de personalidad, actitudes, valores, conocimientos, aptitudes y habilidades (Mitrani et al. 1992). 
La adopción del enfoque de competencias permite focalizar los esfuerzos de todas las personas hacia la consecución de resultados; contribuye a la predicción del comportamiento futuro de las personas sobre la base de su comportamiento pasado; y facilita la comparación entre el perfil de exigencias del puesto y el perfil competencial de las personas.

La Organización Internacional del Trabajo (OIT) define la competencia como "la capacidad de articular y movilizar condiciones intelectuales y emocionales en términos de conocimientos, habilidades, actitudes y prácticas, necesarias para el desempeño de una determinada función o actividad, en forma eficiente, eficaz y creativa, conforme a la naturaleza del trabajo. La capacidad productiva de un individuo se define y mide en términos de desempeño real y demostrado en determinado contexto de trabajo y no resulta solo de la instrucción sino de la experiencia en situaciones concretas de ejercicio ocupacional". Criterios complementarios señalan que los componentes que determinan las competencias son conocimientos, habilidades y destrezas, actitudes $\mathrm{y}$ valores.

La identificación de las competencias propias de un puesto de trabajo constituye una de las primeras acciones que deben cubrirse en el ámbito de la gestión de los recursos humanos, y que forma parte de la descripción de los puestos de trabajo de la organización. Esa descripción del perfil competencial propio de un puesto de trabajo será precisamente la referencia común a la hora de llevar a cabo la evaluación de competencias.

Los conocimientos adquiridos a través de la educación formal constituyen una fortaleza menor entre el personal de administración y guías, y una debilidad menor entre el personal de las áreas de apoyo. Las habilidades y destrezas de los trabajadores son una fortaleza mayor entre los guías y administradores; en cambio, constituyen una fortaleza menor entre el personal de las áreas de apoyo. Los valores como la conservación del medio ambiente, la honestidad, la responsabilidad y el respeto constituyen una fortaleza mayor entre todos los trabajadores; el cumplimiento del trabajo, la equidad y la solidaridad son una fortaleza menor. Las actitudes son una fortaleza mayor en aspectos como conservación, responsabilidad, colaboración, laboriosidad y equidad; es una fortaleza menor en el aspecto de honestidad.

\section{CONCLUSIONES}

Se ha encontrado:

1. La investigación demuestra que del total de estudiantes analizados, el 54,17\% tienen una percepción buena sobre las competencias básicas, el $67,71 \%$ opinan que son buenas las competencias genéricas y el 58,33\% opinan que son buenas las competencias específicas.

2. La investigación demuestra que del total de estudiantes analizados, el 40,63\% opina que son buenas las competencias cognoscitivas y el $58,3 \%$ opina que son buenas las habilidades y destrezas.

3. La investigación demuestra que del total de estudiantes analizados el 59,38\% opina que son buenas las competencias laborales, el $50 \%$ opinan que es regular, y el $48,96 \%$ opina que es buena la formación de los profesionales en turismo.

4. La investigación demuestra que el desarrollo de las competencias básicas y la formación en competencias cognoscitivas del perfil se relacionan significativamente, concluyendo que existe una correlación moderada positiva y significativa $(0,39)$ entre el desarrollo de las competencias básicas y la formación en competencias cognoscitivas del perfil de los profesionales en turismo.

5. La investigación demuestra que el desarrollo de las competencias básicas y la formación en habilidades y destrezas del perfil se relacionan significativamente, concluyendo que existe una correlación moderada positiva y significativa $(0,41)$ entre el desarrollo de las competencias básicas y la formación en habilidades y destrezas del perfil de los profesionales en turismo

6. La investigación demuestra que el desarrollo de las competencias genéricas y la formación en competencias cognoscitivas del perfil se relacionan significativamente, concluyendo que existe una correlación moderada positiva y significativa $(0,47)$ entre el desarrollo de las competencias genéricas y la formación en competencias cognoscitivas del perfil de los profesionales en turismo.

7. La investigación demuestra que el desarrollo de las competencias genéricas y la formación en habilidades y destrezas del perfil se relacionan significativamente, concluyendo que existe una correlación moderada positiva 
y significativa $(0,61)$ entre el desarrollo de las competencias genéricas y la formación en habilidades y destrezas del perfil de los profesionales en Turismo.

8. La investigación demuestra que el desarrollo de las competencias específicas y la formación en competencias cognoscitivas del perfil se relacionan significativamente, concluyendo que existe una correlación moderada positiva y significativa $(0,49)$, entre el desarrollo de las competencias específicas y la formación en competencias cognoscitivas del perfil de los profesionales en turismo.

9. La investigación demuestra que el desarrollo de las competencias específicas y la formación en habilidades y destrezas del perfil se relacionan significativamente, concluyendo que existe una correlación moderada positiva y significativa $(0,59)$ entre el desarrollo de las competencias específicas y la formación en habilidades y destrezas del perfil de los profesionales en turismo.

\section{BIBLIOGRAFÍA}

Bernal, C. (2000). Metodología de la investigación para administración y economía. Pearson, Colombia.

Capella, J. (2002). Desafíos del Foro Educativo en la hora actual. Lautrec SRL., Lima.

Castro, E. (1999). La metodología de la investigación científica. Universidad Cristiana María Inmaculada, Lima.

Chacaltana, J.; Saravia, C.; y Rosas, S. (2011). Trayectorias de vida y empleabilidad. Fondo de Población de las Naciones Unidas (UNFPA), Lima.

Diario Oficial El Peruano. (1993). Constitución Política del Perú de 1993. Empresa Editora Perú, Lima.

García, J. y Pérez, M. (2008). "El grado en turismo: un análisis de las competencias profesionales”. En: Cuadernos de Turismo. $N^{\circ} 21$. Enerojunio 2008. Universidad de Murcia, España.

Garrigós y Narangajavana. (2006). Título. Editorial, Ciudad.

Gómez, S. (2010). La experiencia de un diseño curricular en turismo basado en un modelo por competencias profesionales. Universidad de Guadalajara, México.
Gómez, V. (2009). "Desempeño laboral del licenciado en turismo de la Universidad de Lunar, de Oriente, según la opinión del sector empresarial turístico privado de la Isla Margarita, Estado de Nueva Esparta". En: Gestión Turística. №11. Junio del 2009.

Hernández, B. (2010). "Formación universitaria de turismo en el Estado de Bolívar". Tesis doctoral. Decanato de Investigación y Post Grado de la Universidad Fermín Toro, Venezuela.

Julca, E. (2011). Evaluación de factores básico de competencias de emprendimiento empresarial en los estudiantes de turismo. El caso de la Universidad de San Martín de Porres, Lima (Perú). Universidad de Salamanca, España.

Kerlinger, F. (1982). Investigación del comportamiento. Técnicas y metodología. Editorial Interamericana, México.

Ley $\mathrm{N}^{\circ} 23733$, Ley Universitaria.

Ley №28044, Ley General de Educación.

Lillo, A.; Ramón, A.; Sevilla, M. (2006). Un marco de análisis del capital humano en turismo. Universidad de Alicante, Departamento de Análisis Económico Aplicado, España.

Martens. (1993). Título. Editorial, Ciudad.

Martínez, J. (2012). "La competencia de empleabilidad de los futuros profesionales del turismo”. En: Revista TUR y DES. Vol. 5, $\mathrm{N}^{\circ} 12$. Junio del 2012.

Mejía, E. (2005). Metodología de la investigación. UNMSM - Unidad de Post Grado de la Facultad de Educación, Lima.

Mitrani et al. (1992). Título. Editorial, Ciudad.

Peñaloza, W. (1997). "Título de artículo”. En: Ministerio de Educación y Electroperú. (1997). Política educativa y tecnología educativa. Ministerio de Educación y Electroperú, Lima.

Robalino, G. (2012). Plan de capacitación por competencias laborales para prestadores de servicios turísticos del Cantón Pillaro, Parroquia San Miguelito. Universidad de Especialidades Turísticas, Ecuador.

Rodríguez, J. (2009). Índices de empleabilidad de los jóvenes. Fundación Carolina CEALCI; Centro de Investigaciones Sociológicas, Económicas, Políticas y Antropológicas (CISEPA); Pontificia Universidad Católica del Perú, Lima. 
Rossi, E. (2005). Construcción y evaluación curricular en la universidad. Editorial, Ciudad.

Rychen y Salgnik. (2001). Título. Editorial, Ciudad.

Rychen y Salgnik. (2003). Título. Editorial, Ciudad.

Tito, P. (2012). "Gestión por competencias y productividad laboral en empresas del sector confección de calzado en Lima Metropolitana”. Tesis para obtener el grado académico de doctor en Ciencias Administrativas. Universidad Nacional Mayor de San Marcos.

Torres, R. y Merino, J. (2010). La ejecución real del Plan Curricular y el uso de medios y materiales en el mejoramiento del rendimiento académico de los estudiantes de la especialidad de Administración de la Facultad de Ciencias Administrativas y Turismo de la Universidad Nacional de Educación (UNE). Informe de investigación. Universidad Nacional de Educación - Enrique Guzmán y Valle - La Cantuta, Vicerrectorado Académico, Dirección del Instituto de Investigación, Lima.
Universidad Nacional Mayor de San Marcos. (2006). Plan de Estudios. Facultad de Ciencias Administrativas, Programa de la EAP. de Administración de Turismo, Lima.

Universidad Nacional Mayor de San Marcos. (2010). Plan Estratégico institucional 2006-2011. Universidad Nacional Mayor de San Marcos, Lima.

Vásquez, M. (2010). "La formación de las competencias de los alumnos de licenciatura en turismo del IPN respecto a los hoteles de la zona metropolitana”. Tesis para obtener el grado de Maestría en Ciencias de la Administración y Desarrollo de la Educación. Sección de Estudios de Post Grado e Investigación de la Escuela Superior de Comercio y Administración del Instituto Politécnico Nacional.

Walingree, N. (2011). Avances en la construcción del conocimiento del turismo: pensando la disciplina del turismo desde una perspectiva integral. Universidad Nacional de Quilmes, Argentina.

Yañiz. (2006). Título. Editorial, Ciudad. 